\title{
Relationship between Hallux Valgus Severity and 3D Ground Reaction Force in Individuals with Hallux Valgus Deformity during Gait
}

\author{
Yong-Wook Kim, PT, $\mathrm{PhD}^{\dagger}$
}

Department of Physical Therapy, College of Medical Sciences, Jeonju University

Received: May 30, 2021 / Revised: June 2, 2021 / Accepted: June 17, 2021

(C) 2021 J Korean Soc Phys Med

\section{| Abstract |}

PURPOSE: This study examined the relationship between the severity of a hallux valgus (HV) deformity and the kinetic three-dimensional ground reaction force (GRF) through a motion analysis system with force platforms in individuals with a HV deformity during normal speed walking.

METHODS: The participants were 36 adults with a HV deformity. The participants were asked to walk on a $6 \mathrm{~m}$ walkway with 40 infrared reflective markers attached to their pelvic and lower extremities. A camera capture system and two force platforms were used to collect kinetic data during gait. A Vicon Nexus and Visual3D motion analysis software were used to calculate the kinetic GRF data.

RESULTS: This research showed that the anterior maximal force that occurred in the terminal stance phase during gait had a negative correlation with the $\mathrm{HV}$ angle $(\mathrm{r}=-.762, \mathrm{p}<.01)$. In addition, the $\mathrm{HV}$ angle showed a low negative correlation with the second vertical maximal force $(r=.346, \mathrm{p}<.05)$ and a moderate positive correlation with the late medial maximal

†Corresponding Author : Yong-Wook Kim ptkim@jj.ac.kr, https://orcid.org/0000-0002-0311-9766

This is an Open Access article distributed under the terms of the Creative Commons Attribution Non-Commercial License (http://creativecommons.org/licenses/by-nc/3.0) which permits unrestricted non-commercial use, distribution, and reproduction in any medium, provided the original work is properly cited. force $(\mathrm{r}=.641, \mathrm{p}<.01)$.

CONCLUSION: A more severe HV deformity results in greater abnormal translation of the plantar pressure and a significantly reduced pressure force under the first metatarsophalangeal joint.

Key Words: Force platform, Hallux valgus, Three dimensions

\section{Introduction}

Hallux valgus (HV) is a foot deformity characterized by the outward deviation of the first intermetatarsal and metatarsophalangeal joints and is generally associated with chronic pain on the medial head of the first metatarsal bone [1-3]. Although the exact causes of HV have not yet been identified clearly, previous studies reported that the causes of $\mathrm{HV}$ include abnormal gait pattern, wearing footwear of insufficient length, inheritance of family physical traits, flat feet, and sex [3-5].

Thus far, various therapeutic interventions have been performed for patients with HV. Nonoperative care, such as physical therapy, foot-toe orthoses, and medication, is always the first treatment option for HV deformities [6-8]. Among these options, various foot-toe orthoses that correct the alignment of the first metatarsophalangeal joint are preferred for minor to moderately severe $\mathrm{HV}$, which have 
significant clinical effectiveness [3,7,9].

To the best of the authors' knowledge, few studies have examined the kinetic relationship between the severity of HV deformity and the three-dimensional (3D) ground reaction force (GRF) using a motion analysis system with force plates during gait. Because the first metatarsophalangeal joint in a normal gait pattern acts as an anchor for the forward translation of body weight force during the late stance phase [10], malalignment or a pathologic dysfunction of the first metatarsophalangeal joint could adversely affect the efficient push-off and alter the GRF [11]. An imbalance of the abductor and adductor hallucis muscles could elicit more medial movement of the center of pressure in the first metatarsophalangeal joint during the late stance phase, contributing to a HV deformity [10].

Understanding the kinetic information, such as joint moments and ground reaction force that occur in the joints and segments of the lower extremities during gait in individuals with $\mathrm{HV}$, is very important. Most previous studies reported the plantar pressure distribution or transfer of plantar pressure in patients with HV during gait $[9,10,12]$. Therefore, this study examined the relationship between the kinetic GRF and HV severity through 3D gait analysis in individuals with HV during gait. This study tested the hypothesis that there is a significant correlation between the severity of a HV deformity and 3D GRF during the stance phase.

\section{Methods}

\section{Subjects}

The sample size was calculated based on an estimated effect size derived from Seki et al. [13], who examined the relationship between the degree of the HV deformity and the kinematics. A sample size of 17 was determined to be sufficient to identify a significant correlation. The subjects in this study were thirty-four adults (14 males and 20 females) with a HV deformity. The inclusion criteria
Table 1. General Characteristics of the Subjects $(N=34)$

\begin{tabular}{cc}
\hline Characteristics & Mean \pm SD \\
\hline Gender & Male: 14, Female: 20 \\
Age (years) & $28.43 \pm 6.15$ \\
Height $(\mathrm{cm})$ & $162.73 \pm 8.05$ \\
Weight $(\mathrm{kg})$ & $63.71 \pm 10.18$ \\
Gait speed $(\mathrm{m} / \mathrm{s})$ & $1.323 \pm .172$ \\
Step length $(\mathrm{cm})$ & $133.11 \pm 10.48$ \\
Step width $(\mathrm{cm})$ & $11.92 \pm 3.07$ \\
\hline
\end{tabular}

were a hallux valgus angle of more than $10^{\circ}$ measured using a universal goniometer in both feet and no severe pain of the hallux area or a history of foot surgery $[2,3]$. The mean and standard deviation of hallux valgus angle was $24.4 \pm 9.3^{\circ}$ in the right foot and $26.2 \pm 12.1^{\circ}$ in the left foot. All participants voluntarily participated in this study. They provided written informed consent and fully understood the purpose and experimental procedure. The Institutional Review Board of Jeonju University approved this study (jjIRB-180905-HR-2018-0904). Table 1 lists the general characteristics of the subjects.

\section{Instrumentation and Procedure}

A motion capture system (Vicon Inc., Oxford, England) with eight infrared cameras (model T10) and two force platforms (AMTI, Massachusetts, USA) was used to obtain the 3D kinetic data during gait (Fig. 1). The 3D GRF kinetic data were transferred to the main computer using Nexus 1.8.5 software (Vicon Inc., Oxford, England) and processed to produce the final $\mathrm{c} 3 \mathrm{~d}$ files. A T-frame wand $(7.5 \mathrm{~cm})$ was used to calibrate the motion capture system and used to set the lab X-Y-Z origin for a calibration reference object. The motion capture-sampling rate of the T10 camera was $100 \mathrm{~Hz}$.

Forty reflective markers $(1.4 \mathrm{~cm})$ were attached to both lower extremities and the anterior and posterior superior iliac spines, greater trochanters, femur epicondyles, malleoli, rearfoot, midfoot, and forefoot (Fig. 1). Four cluster refl 


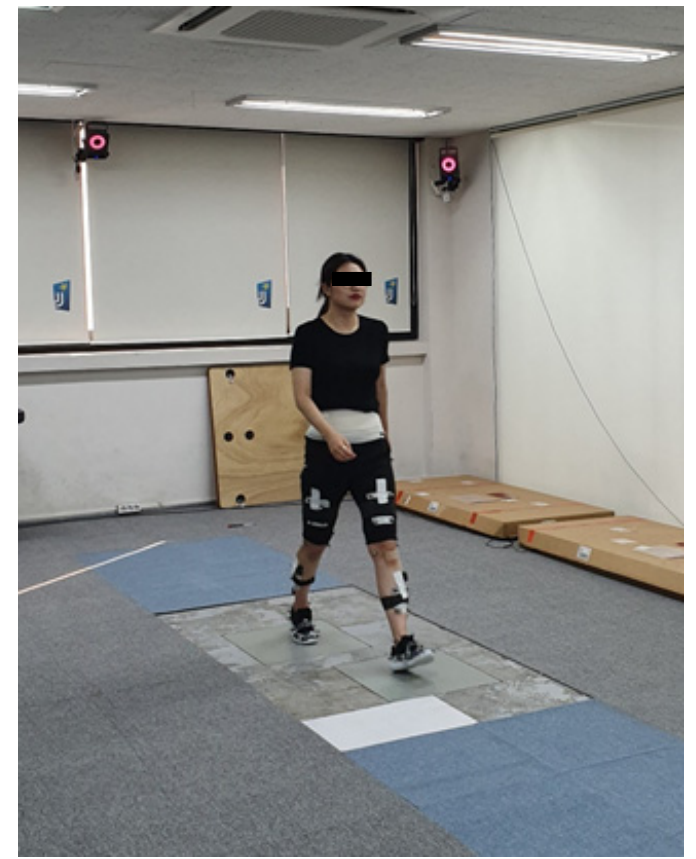

Fig. 1. Dynamic motion capture system with eight infrared cameras and two force platforms to obtain the 3D kinetic data during gait.

ective markers were attached bilaterally to the calf and thigh segments using the six-degrees-of-freedom model [14]. Static calibration capture was used on each subject for 3D GRF analysis during the walking trials to produce a template model for analysis. The calibrated anatomical system technique was used to identify the changes in the kinetic GRF data of the lower extremities while the subjects walked freely. After completing the experimental setup for motion analysis, the subjects were asked to walk along a $6 \mathrm{~m}$ walkway at a comfortable speed. The kinetic GRF data from two force platforms were collected while performing 8 to 10 walking trials.

After collecting the analog GRF data using two force platforms and the Vicon Nexus software program, Visual3D motion analysis software (Visual3D Pro, C-Motion Inc., USA) was used to obtain the final dynamic GRF results and the 3D graph reports of both extremities during the stance phase. The GRF kinetic data were

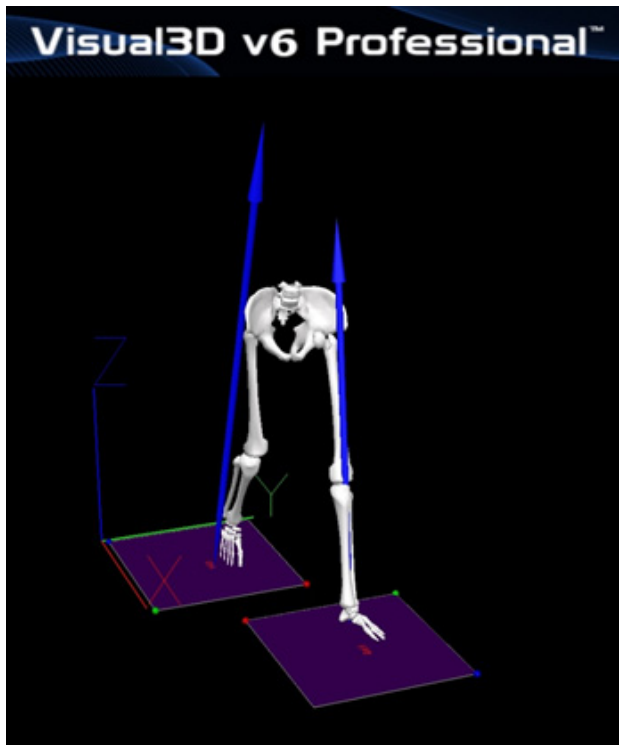

Fig. 2. Visual3D motion analysis of the magnitudes and directions of the GRF of both limbs in the 3D space during gait.

low-pass filtered with a fourth-order Butterworth filter and a cutoff frequency of $15 \mathrm{~Hz}$. Following the right-hand rule for the segment coordinate system axes, the X-Y-Z Cardan sequence was used to define the order of the rotations [14]. Visual3D produced the virtual direction and amplitude of dynamic 3D GRF in laboratory space based on a motion capture system that enabled the relevant 3D GRF to be calculated for statistical analysis during the stance phase in the total gait cycle (Fig. 2).

\section{Data Analysis}

The Kolmogorov-Smirnov test was used to confirm that the kinetic GRF and HV angle data were distributed normally. The Pearson product-moment correlation analysis was used to verify the relationship between the HV angle and the 3D GRF variables. The GRF variables consisted of the posterior maximal force, initial mediolateral maximal force, initial vertical maximal force, midstance vertical minimal force, late vertical maximal force, anterior maximal force, and late mediolateral maximal force 


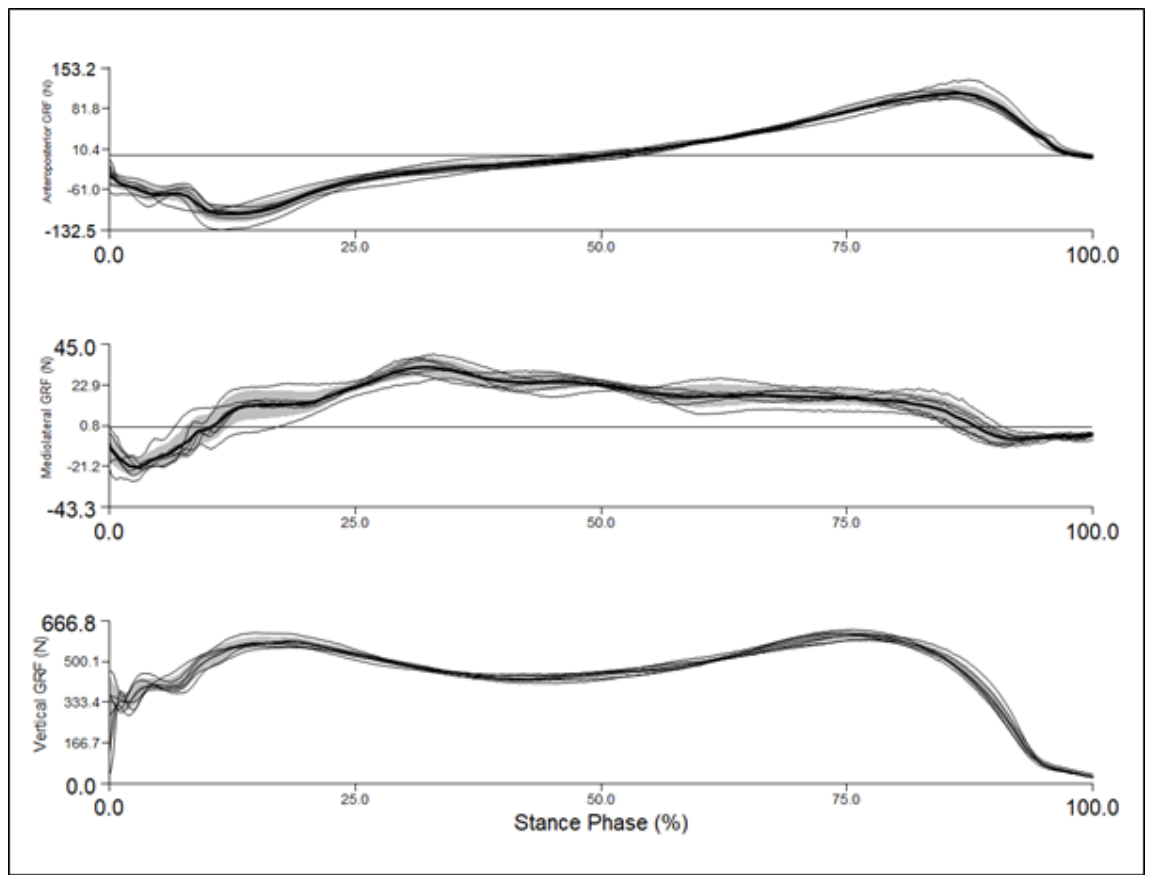

Fig. 3. Three-dimensional ground reaction force variability developed in each plane during the stance phase. The means (thick lines), each trial (thin line), and standard deviation (bands) are shown.

Table 2. Mean \pm SD of the Ground Reaction Force and Hallux Valgus Angle

\begin{tabular}{ccccc}
\hline Gait cycle & Variables (N/kg) & Right foot & Left foot & $\mathrm{p}$ \\
\hline $0-25 \%$ & PMF & $1.79 \pm .33$ & $1.82 \pm .42$ & .442 \\
Stance phase & ILMF & $.62 \pm .01$ & $.64 \pm .02$ & .094 \\
& 1stVMF & $10.58 \pm 1.04$ & $10.46 \pm .94$ & .725 \\
\hline $\begin{array}{c}25-75 \% \\
\text { Stance phase }\end{array}$ & MMVF & $6.58 \pm 1.03$ & $6.75 \pm .97$ & .221 \\
\hline 75-100\% & 2ndVMF & $11.20 \pm 1.21$ & $11.094 \pm 1.01$ & .770 \\
Stance phase & MAF & $2.11 \pm .22$ & $2.12 \pm .19$ & .534 \\
& LMMF & $.57 \pm .15$ & $.485 \pm .22$ & .083 \\
\hline
\end{tabular}

PMF: Posterior maximal force, ILMF: Initial lateral maximal force, VMF: Initial vertical maximal force, MVMF: Midstance vertical minimal force, MAF: Maximal anterior force, LMMF: late medial maximal force, HVA: Hallux valgus angle

developed in each motion plane during the stance phase of gait (Fig. 3). All analyses were conducted using SPSS version 26.0 (IBM Corp., Armonk, NY, USA). Differences were considered significant at the $a=.05$ level.

\section{Results}

Table 2 lists the means and standard deviations of the GRF variables and the HV angles of both feet. No significant differences were observed between the right and 
Table 3. Correlations between the Hallux Valgus Angle and Ground Reaction Force Variables

\begin{tabular}{ccccccccc}
\hline & HVA & PMF & ILMF & 1stVMF & MVMF & 2ndVMF & MAF & LMMF \\
\hline PMF & .052 & 1 & & & & & & \\
ILMF & -.075 & $.788^{* *}$ & 1 & & & & & \\
1 stVMF & -.141 & $.502^{*}$ & $.474^{*}$ & 1 & & & & \\
MVMF & -.124 & -.118 & .067 & $-.492^{*}$ & 1 & & & \\
2ndVMF & $-.346^{*}$ & $.633^{* *}$ & .184 & $.776^{* *}$ & $-.553^{*}$ & 1 & & \\
MAF & $-.762^{* *}$ & $.811^{* *}$ & .104 & $.713^{* *}$ & -.290 & $.794^{* *}$ & 1 & \\
LMMF & $.641^{* *}$ & $.674^{* *}$ & $.557^{*}$ & .304 & .071 & $.509^{*}$ & $.812^{* *}$ & 1 \\
\hline${ }^{*}$
\end{tabular}

${ }^{*} \mathrm{p}<.05,{ }^{* *} \mathrm{p}<.01$

PMF: Posterior maximal force, ILMF: Initial lateral maximal force, VMF: Initial vertical maximal force, MVMF: Midstance vertical minimal force, MAF: Maximal anterior force, LMMF: late medial maximal force, HVA: Hallux valgus angle

left feet of all GRF variables and HV angles $(\mathrm{p}>.05)$ (Table 2).

The correlations between the HV angle and some 3D GRF variables showed significant differences (Table 3). The anterior maximal force in the terminal stance phase during gait showed a highly negative correlation with the $\mathrm{HV}$ angle $(\mathrm{r}=-.762, \mathrm{p}=.000)$. In addition, the HV angle showed a low negative correlation with the second vertical maximal force $(\mathrm{r}=-.346, \mathrm{p}=.025)$ and a moderate positive correlation with the late medial maximal force $(\mathrm{r}=.641$, $\mathrm{p}=.002$ ). On the other hand, there was no significant correlation between the $\mathrm{HV}$ angle and the other GRF variables $(p>.05)($ Table 3$)$. The result showed a significant correlation among the various GRF variables. The posterior maximal force showed a significant moderate or high correlation with all the GRF variables except for the midstance vertical minimal force, and the initial lateral maximal force showed a significant correlation with the first vertical force $(\mathrm{r}=.474, \mathrm{p}=.008)$ and the late medial maximal force $(r=.557, p=.004)($ Table 3).

\section{Discussion}

This study examined the relationship between the HV angle and various GRF values obtained during the free walking trials using $3 \mathrm{D}$ motion analysis in individuals with
HV. The HV angle was significantly negatively correlated with most of the 3D GRF variables that occurred at certain sections of the stance phase during free walking. In particular, a strong negative correlation was noted between the HV angle and the anterior maximal force occurring at $75 \%$ to $100 \%$ of the stance phase during gait $(r=-.762)$. This result means that the push-off or toe-off force generated from the ground contacting the foot in the terminal stance phase during walking decreases as the HV angle increases. Among the metatarsophalangeal and intertarsal joints, the first metatarsophalangeal joint is the largest. It plays an important role as a pivot for weight-bearing transfer of the forefoot during the late stance phase $[10,15]$. In addition, it provides an attachment site for various muscles, such as the adductor hallucis, abductor hallucis, flexor hallucis brevis muscles, and plantar fascia, which support the longitudinal foot arch of the medial foot area [16]. Therefore, a HV deformity may cause a problem in supporting the medial longitudinal arch of the foot and trigger pes planus $[17,18]$. Although these results could not be compared with previous studies directly, some studies examined the relationship between plantar pressure distribution and $\mathrm{HV}$ deformity using a treadmill with an electric pressure sensor mat [10] or a simple mat transducer platform during gait [12]. The second and third 
metatarsophalangeal joints and the fourth and fifth toes showed a significantly higher pressure distribution than in healthy control feet [10]. Compared to normal control subjects, patients with a HV deformity demonstrated a significant medial plantar pressure peak and mean pressures under the first, second, and third metatarsal heads [10]. In addition, patients with $\mathrm{HV}$ deformity showed a significantly lower range of ankle joint motion during gait than the control group [19]. Therefore, the anterior repulsive force that occurred to the forefeet and toes from the ground in the late stance phase plays a critical role for activities of the foot and ankle joints in normal gait patterns and daily activities, such as standing balance [20,21].

These results showed that the HV severity has a significant positive correlation with the late medial maximal force in $75 \%$ to $100 \%$ of the stance phase. A previous study reported the average maximal pressure differences of all metatarsal heads of the foot of 15 subjects with HV and 30 subjects in a normal control group [22]. The result showed a medial shift in maximal pressure in the HV group with a significant decrease in plantar pressure distribution under the lateral metatarsal heads. Similarly, Hoffmann et al. [10] used a pedobarographic-instrumented treadmill to compare the mean maximal plantar pressure differences of all metatarsal heads and toes of 36 patients with a unilateral HV deformity. They reported higher pressures in the HV feet beneath the second and third metatarsal heads and the fourth and fifth toes in the control feet. Compared to previous studies, this study provided evidence that as the severity of the HV deformity increases, a larger abnormal shift of the plantar pressure occurs and the pressure force under the hallux is reduced significantly.

The results of this study showed significant correlations among most of the GRF variables. Few previous studies verified the correlation according to HV severity by analyzing the specific variables of the 3D GRF during gait. Hence, a direct comparison with the results of this study was not possible. On the other hand, because the GRF variable generated during gait is calculated as a vector value in 3D space, a change in the magnitude and direction of the force in a specific motion plane will affect the other motion planes. Therefore, further studies will be needed to verify the biomechanical characteristics and interactions of the 3D GRF variables that occur in patients with HV during walking.

This study had some limitations. Although the motion analysis system setup has many advantages, gait in such a laboratory environment affects the gait patterns and GRF data compared to general walking environments. In addition, this study examined healthy participants, who were mostly younger than the general age. Therefore, it is difficult to generalize the study results to all patients with a HV deformity. The analyses of this study focused only on the maximal GRF that occurred in three different motion planes. Considering the difficulty of the analysis, the other kinetic variables, such as the joint moment and power, were not calculated. Further studies will be needed to investigate the relationship between the HV severity and the various biomechanical changes in patients with a HV deformity.

\section{Conclusion}

This study examined the relationship between the HV angle and 3D dynamic GRF variables during walking in 34 individuals with a HV deformity. The Pearson productmoment correlation analysis revealed a significant correlation between the $\mathrm{HV}$ angle and the posterior maximal force in the late stance phase. In addition, the HV angle showed a negative correlation with the second vertical maximal force and a positive correlation with the late medial maximal force. These results indicate that the extent of the abnormal translation of plantar pressure is related to the severity of the HV deformity. Moreover, the pressure force under the first metatarsophalangeal joint is reduced significantly with increasing severity of the HV deformity. Therefore, a more 
aggressive early evaluation and treatment will be needed in clinical practice to prevent and manage HV deformities.

\section{References}

[1] Sun PC, Shih SL, Chen YY, et al. Evaluation of patients with hallux valgus wearing a 3d-printed orthosis during walking. Appl Sci. 2021;11(3):1275.

[2] Coughlin MJ, Jones CP. Hallux valgus: demographics, etiology, and radiographic assessment. Foot Ankle Int 2007;28(7):759-77.

[3] Kim YW. Effects of hallux valgus orthoses on ground reaction force using a 3D motion analysis in individuals with hallux valgus deformity. Phys Ther Korea. 2020; 27(4):227-32.

[4] Steinberg N, Siev-Ner I, Zeev A, et al. The association between hallux valgus and proximal joint alignment in young female dancers. Int J Sports Med. 2015;36(1):67-74.

[5] Klein C, Groll-Knapp E, Kundi M, et al. Increased hallux angle in children and its association with insufficient length of footwear: a community based cross-sectional study. BMC Musculoskelet Disord. 2009;10:159.

[6] Coughlin MJ. Hallux valgus in men: effect of the distal metatarsal articular angle on hallux valgus correction. Foot Ankle Int. 1997;18(8):463-70.

[7] Tang SF, Chen CP, Pan JL, et al. The effects of a new foot-toe orthosis in treating painful hallux valgus. Arch Phys Med Rehabil. 2002;83(12):1792-5.

[8] Kim MH, Yi CH, Weon JH, et al. Effect of toe-spread-out exercise on hallux valgus angle and cross-sectional area of abductor hallucis muscle in subjects with hallux valgus. J Phys Ther Sci. 2015;27(4):1019-22.

[9] Doty JF, Alvarez RG, Ervin TB, et al. Biomechanical Evaluation of Custom Foot Orthoses for Hallux Valgus Deformity. J Foot Ankle Surg. 2015;54(5):852-5.

[10] Hofmann UK, Götze M, Wiesenreiter K, et al. Transfer of plantar pressure from the medial to the central forefoot in patients with hallux valgus. BMC Musculoskelet Disord.
2019;20(1):149.

[11] Rouhani H, Favre J, Crevoisier X, et al. Ambulatory assessment of $3 \mathrm{D}$ ground reaction force using plantar pressure distribution. Gait Posture. 2010;32(3):311-6.

[12] Bryant A, Tinley P, Singer K. Plantar pressure distribution in normal, hallux valgus and hallux limitus feet. The Foot. 1999;9(3):115-9.

[13] Seki H, Miura A, Sato N, et al. Correlation between degree of hallux valgus and kinematics in classical ballet: A pilot study. PLoS One. 2020;15(4):e0231015.

[14] Collins TD, Ghoussayni SN, Ewins DJ, et al. A six degrees-of-freedom marker set for gait analysis: repeatability and comparison with a modified Helen Hayes set. Gait Posture. 2009;30(2):173-80.

[15] Hughes J, Clark P, Klenerman L. The importance of the toes in walking. J Bone Joint Surg Br. 1990;72(2): 245-51.

[16] Cael C. Functional anatomy: Musculoskeletal anatomy, kinesiology, and palpation for manual therapist. USA. Lippincott Willians \& Wilkins. 2010.

[17] King DM, Toolan BC. Associated deformities and hypermobility in hallux valgus: an investigation with weightbearing radiographs. Foot Ankle Int. 2004;25(4): 251-5.

[18] Kalen V, Brecher A. Relationship between adolescent bunions and flatfeet. Foot Ankle. 1988;8(6):331-6.

[19] Klugarova J, Janura M, Svoboda Z, et al. Hallux valgus surgery affects kinematic parameters during gait. Clin Biomech (Bristol, Avon). 2016;40:20-26.

[20] Ballas R, Edouard P, Philippot R, et al. Ground-reactive forces after hallux valgus surgery: comparison of Scarf osteotomy and arthrodesis of the first metatarsophalangeal joint. Bone Joint J. 2016;98-B(5):641-6.

[21] Barbee CE, Buddhadev HH, Chalmers GR, et al. The effects of hallux valgus and walking speed on dynamic balance in older adults. Gait Posture. 2020;80:137-42.

[22] Plank MJ. The pattern of forefoot pressure distribution in hallux valgus. The Foot. 1995;5:8-14. 